\title{
Potential impact of population screening for prenatal diagnosis of congenital heart disease
}

\author{
Seamus Cullen, Gurleen K Sharland, Lindsey D Allan, Ian D Sullivan
}

\begin{abstract}
The potential impact of prenatal screening for the detection of congenital heart disease (CHD) was assessed by prospective analysis of 428 consecutive infant admissions to a supraregional centre; $28(6.5 \%)$ did not have CHD and were excluded from analysis. Of the 400 cases with CDH, 396 (99\%) underwent fetal ultrasonography but scanning was performed only before 18 weeks' gestation in $200(50 \%)$. One hundred and forty nine (37\%) of all cardiac abnormalities and $149 / 283(53 \%)$ of severe abnormalities were considered to be detectable prenatally in a screening echocardiographic four chamber view had this technique been used. Prenatal diagnosis of severe CHD actually occurred in only eight $(2 \%)$ cases and was after 30 weeks' gestation in all. There were 181/347 (52\%) of all mothers and 177/253 (70\%) of the subgroup with severe abnormalities who expressed an opinion volunteered their preference for termination of pregnancy if mid-trimester diagnosis had been available.
\end{abstract}

Mid-trimester detection of congenital heart disease rarely occurs at present despite fetal ultrasound scanning in almost all pregnancies. More than half of all severe congenital heart defects seen in infancy are potentially detectable by screening. Major training at primary scan level and modification of the timing of existing fetal anomaly scanning would be required for a screening programme to be effective.

Mid-trimester screening for the detection of fetal anomalies, including congenital heart disease (CHD), is advocated in the UK. ${ }^{1}$ Fetal echocardiography is an accurate method for the diagnosis of cardiac defects prenatally. ${ }^{2}$ A survey in France demonstrated the feasibility of mid-trimester screening for structural heart disease using a transverse ultrasound section through the fetal thorax to provide a four chamber echocardiographic view of the fetal heart. ${ }^{3}$ This has been confirmed in a small number of obstetric units in the UK. ${ }^{45}$ An increasing number of cases of CHD have been detected prenatally because a heart abnormality was suspected on the routine obstetric scan (fig 1).

This study was undertaken to determine the potential impact on paediatric cardiology practice if a similar antenatal screening programme for CHD was extended to the whole population.

\section{Methods}

British born neonates and infants admitted to the cardiac unit of the Hospital for Sick Children between September 1989 and March 1991 were assessed. A questionnaire was administered (usually by SC, less frequently by IDS) to their mothers within one week of admission. Both parents were present in approximately half of the interviews. Obstetric and birth history, cardiac diagnosis and other congenital abnormalities, acute management, timing of antenatal ultrasound scanning at the local hospital, and the detection of cardiac and extracardiac anomaly prenatally were recorded. Family history of CHD was determined. Parental perception of information offered concerning possible cardiac anomalies at the time of routine prenatal ultrasound scanning at the local hospital was ascertained by asking 'was any comment made about the heart?' In addition, mothers were asked if prior knowledge of a fetal cardiac anomaly would have been helpful, but not specifically about termination of pregnancy.

The normal four chamber view of the fetal heart has characteristic features. The heart occupies about one third of the area of the fetal thorax in transverse section, the two atria are of similar size, the two ventricles are of similar size, and two separate atrioventricular valves meet at the junction of the atrial septum and ventricular septum to form an offset cross (fig 2). For the purposes of this analysis, it was assumed that the four chamber view of the fetal heart would detect fetal

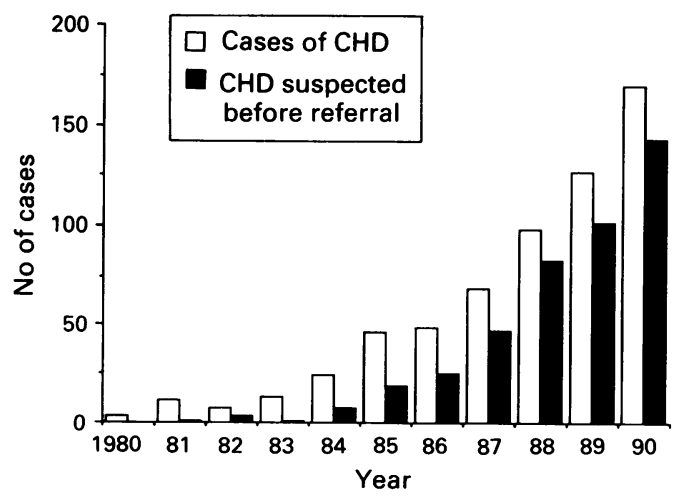

Figure 1 The number of cases of congenital heart defects (CHD) detected annually 1980 to 1990 and the proportion of these referred for assessment because of a suspected abnormality of the fetal heart on the routine ultrasound scan. 


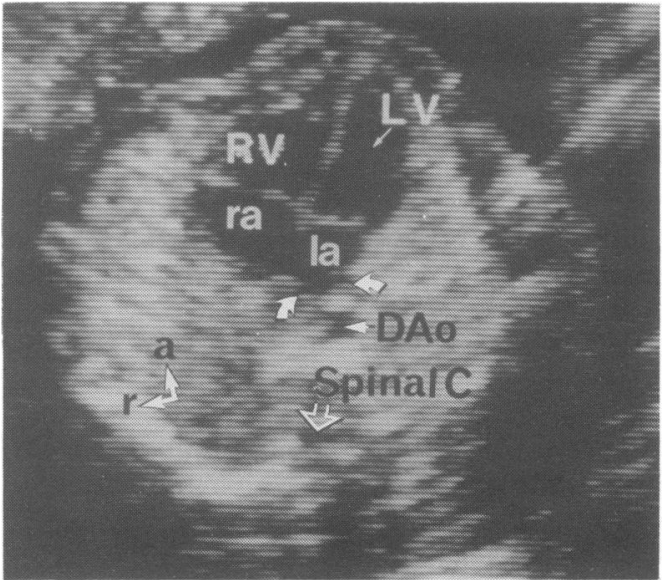

Figure 2 The normal four chamber view of the fetal heart, showing anterior (a), descending aorta (DAo), left atrium (a), left ventricle ( $L V)$, right (r), right atrium ( $r a)$, right ventricle (RV), and spinal cord (Spinal $C)$.

cardiac anomalies characterised by marked cardiomegaly, obvious disparity between the size of the two atria and/or that of the two ventricles, or major abnormalities of the atrioventricular connection. It was assumed that this single screening view would not detect abnormalities likely to be characterised by less obvious abnormalities of the four chamber view, ventricular septal defects, or even major abnormalities of the arterial pole of the heart such as complete transposition or tetralogy of Fallot.

Cardiac defects were graded arbitrarily as non-severe or severe. Non-severe abnormalities were lesions where either no intervention is likely to be required or where a single surgical or non-surgical intervention with anticipated mortality less than 5\% may be necessary. Severe CHD was defined as lesions requiring emergency or elective repair where the perioperative risk nationally was estimated to be greater than $5 \%$ and

Spectrum of congenital heart defects

\begin{tabular}{|c|c|c|c|}
\hline Non-severe $(n=117)$ & & Severe $(n=283)$ & \\
\hline $\begin{array}{l}\text { VSD } \\
\text { Coarctation } \\
\text { PDA } \\
\text { ASD } \\
\text { Pulmonary stenosis } \\
\text { Tricuspid dysplasia } \\
\text { Pulmonary artery stenoses }\end{array}$ & $\begin{array}{r}50 \\
31 \\
25 \\
5 \\
3 \\
2 \\
1\end{array}$ & $\begin{array}{l}\text { Tetralogy of Fallot } \\
\text { AVSD } \\
\text { TGA+VSD } \\
\text { Pulmonary atresia } \\
\text { Pulmonary atresia, IVS } \\
\text { HLHS } \\
\text { Coarctation, VSD } \\
\text { Aortic interrruption } \\
\text { Tricuspid atresia } \\
\text { Arterial trunk } \\
\text { Double inlet ventricle } \\
\text { Critical pulmonary stenosis } \\
\text { Critical aortic stenosis } \\
\text { Complex TGADORV } \\
\text { Ebstein's anomaly } \\
\text { TAPVC } \\
\text { cTGA, VSD } \\
\text { Coarctation, LVOTO } \\
\text { Aortic stenosis } \\
\text { Aortopulmonary window } \\
\text { Cardiomyopathy } \\
\text { Cardiac tumours }\end{array}$ & $\begin{array}{l}54 \\
53^{*} \\
44 \\
17 \\
16^{*} \\
15^{*} \\
11^{*+} \\
11^{*} \\
8^{*} \\
8 \\
7^{*} \\
7^{*} \\
2^{* \ddagger} \\
7^{*}+ \\
5^{*} \\
5 \\
3^{*} \\
3^{*} \\
2 \\
2 \\
2 \\
1^{*}\end{array}$ \\
\hline
\end{tabular}

ASD, atrial septal defect; AVSD, atrioventricular septal defect; cTGA, congenitally corrected transposition of the great arteries; DORV, double outlet right ventricle; HLHS, hypoplast obstruction; PDA, patent ductus arteriosus; TGA, transposition of the great arteries; TAPVC total anomalous pulmonary venous connection; VSD, ventricular septal defect. $*$ Detectable on fetal four chamber view.

tIncludes one patient with aortopulmonary window.

$\neq$ Lesions with marked ventricular disproportion.

fIncludes one patient with TAPVC.

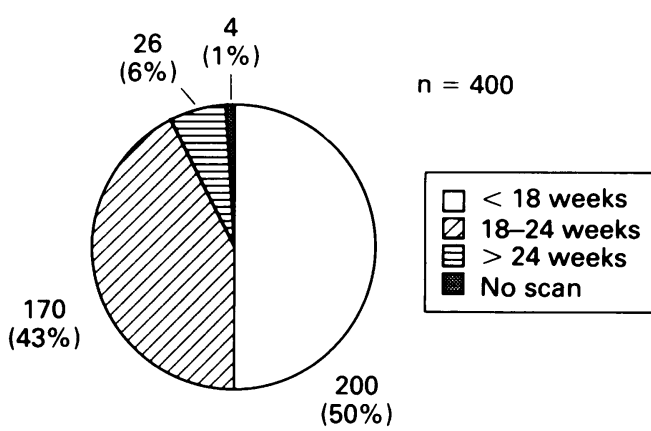

Figure 3 Gestational age at initial fetal ultrasound scan.

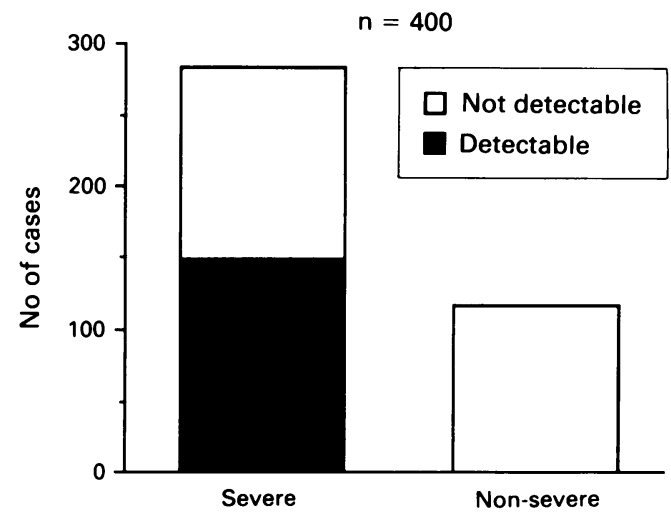

Figure 4 The proportions of non-severe and severe congenital heart defects detectable on a single four chamber screening of the fetal heart.

abnormalities which would inevitably require multiple operations to ensure long term survival. Also included in this category were lesions for which survival would depend on palliative surgery or heart transplantation. The necessity for emergency neonatal repair was not, of itself, a determinant of category of severity. Hence, isolated coarctation of the aorta with normal intracardiac anatomy was classified as non-severe whereas coarctation with a large ventricular septal defect was classified as severe.

\section{Results}

Twenty eight neonates did not have structural heart disease and were excluded from further analysis. Of the 400 neonates and infants with CHD, 396 had been examined by fetal ultrasound scanning at the local hospital. A single scan had been performed before 18 weeks' gestation in $200(50 \%)$, a further 170 $(43 \%)$ underwent initial scanning between 18 and 24 weeks' gestation, whereas the initial scans in the remaining $26(7 \%)$ were performed after 24 weeks (fig 3).

The cardiac abnormalities were not severe in $117(29 \%)$ and severe in $283(71 \%)$ cases (table). Of those with severe heart defects, $149(53 \%)$ could have been detected on a screening four chamber view (table, fig 4). In only eight $(2 \%)$ cases was there a prenatal diagnosis of cardiac anomaly. The diagnosis was made in the third trimester in all of these (fig 5). These eight mothers had been referred for detailed fetal echocardiography because of 


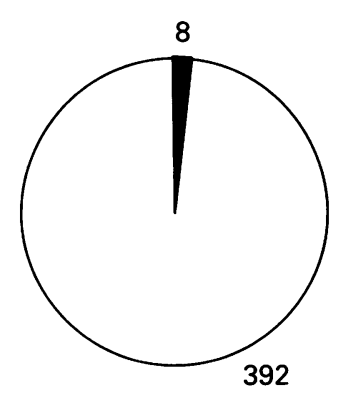

$n=400$

口 > 28 weeks - Not detected

Figure 5 The prenatal detection of congenital heart defects. suspected cardiac $(n=6)$ or extracardiac $(n=2)$ abnormality on routine antenatal scanning.

There were 193 mothers who believed that they had been reassured about the fetal heart at the time of routine scanning. Among these were 90 cases in whom the cardiac anomaly would have been detectable on a four chamber view. In the remainder no specific reference to the fetal heart had been made. There were $181(45 \%)$ mothers who volunteered their preference for termination of pregnancy had they been aware of the diagnosis, $166(42 \%)$ who indicated that they would have continued the pregnancy even though aware of fetal cardiac anomaly, whereas $53(13 \%)$ did not offer an opinion clearly one way or the other. From the subgroup of 283 mothers of babies with severe congenital heart defects, $177(62 \%)$ would have opted for termination of pregnancy $76(27 \%)$ would have continued the pregnancy, and the choice of the remaining $30(11 \%)$ was not ascertained.

A family history of CHD was present in 10 $(2.5 \%)$ with a previously affected sibling in six and a parent with CHD in four. Of these 10 infants, four had severe CHD potentially detectable on screening. In addition, 13 babies (all with severe CHD) had an extracardiac anomaly which might have been detected on antenatal ultrasound scanning: diaphragmatic hernia $(n=5)$, hydronephrosis $(n=4)$, omphalocele $(n=2)$, renal cysts $(n=1)$, and absent kidney $(n=1)$. Only six of these extracardiac anomalies were detected prenatally, these led to the prenatal detection of severe CHD after referral for detailed fetal echocardiography in two, both beyond 32 weeks' gestation. Figure 6 shows the potential prenatal detection of cases of severe CHD by population screening, compared with strategies where pregnancies deemed to be at high risk for CHD, because of a family history or because of the detection of non-cardiac anomaly on fetal scanning, are referred for detailed fetal echocardiography. By far the highest proportion of cases is potentially detectable by population screening.

\section{Discussion}

THE CASE FOR SCREENING

Advocates of population screening for fetal anomaly have recommended that a detailed fetal anomaly scan be offered at district hospitals at 18 to 20 weeks' gestation. ${ }^{1}$ Selected cases might be referred to regional or supraregional centres. In our study group, almost all mothers received fetal ultrasound examination at their local hospital. Prenatal diagnosis of CHD occurred in a tiny minority of this group, and even then was in the third trimester. Most mothers of babies with severe CHD who offered an opinion stated that they would have elected termination of pregnancy if they had been aware of the cardiac anomaly sufficiently early.

The highest yield of positive scans in referral centres is in the group referred with a suspected fetal cardiac abnormality on the four chamber view on the routine scan in the district hospital (fig 1). Obstetric ultrasonographers from one region who were taught to screen the fetal heart detected 69\% of heart abnormalities which it was considered could be visualised on the four chamber view. ${ }^{5}$ The very low rate of detection of heart abnormalities in our study group was contributed to by prenatal diagnosis and subsequent termination of pregnancy in some cases. Of the 169 cases of CHD diagnosed prenatally in 1990 (fig 1), 23 were from obstetric units in centres which would normally refer infants with suspected CHD to the Hospital for Sick Children. The pregnancy was terminated in 12 of these. It is not appropriate to extrapolate from these numbers directly to the postnatal series because of the different spectrum of abnormalities and high rates of intrauterine death and very early neonatal death in series of heart abnormalities diagnosed prenatally. ${ }^{2}$ Nevertheless, it is likely that at least 10 cases of severe severe CHD which would otherwise have appeared in the postnatal group were prevented. The previous strategy in referral centres was to assess in detail designated 'high risk' pregnancies, usually those with a family history of CHD. This approach would not be very rewarding in terms of prenatal diagnosis in new cases in the longer term (fig 6). However, the psychological value afforded to parents with a previously affected child by exclusion of recurrence of severe CHD by detailed fetal echocardiography should not be underestimated.

CHD occurs in $8 / 1000$ live births, about four of which will be severe abnormalities. Our data support the previous suggestion that four chamber screening potentially would detect about two cases of severe CHD per 1000 live births. ${ }^{2}$ There are about 700000 live births annually in the UK. ${ }^{6}$ Consequently, an effective national screening programme based on the four chamber view would potentially detect about 1400 cases of severe

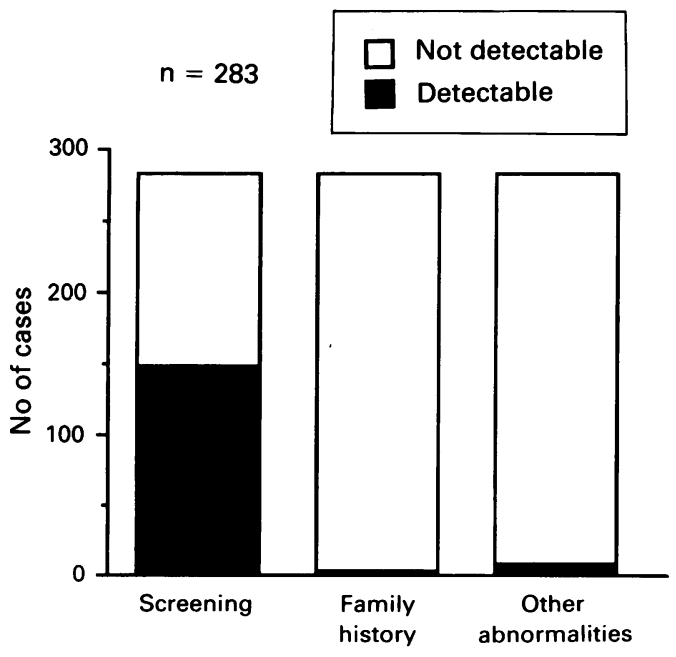

Figure 6 The proportion of cases of severe congenital heart defects detectable by population screening, compared with detailed assessment of the fetal heart in high risk pregnancies (those with a family history of CHD) or those in whom an extracardiac abnormality is detected. 
CHD annually over and above those cases destined for spontaneous intrauterine or very early neonatal death.

\section{LIMITATIONS}

If population fetal anomaly screening is to implemented, current practice and attitudes ${ }^{7}$ require modification. The likelihood of detection of fetal cardiac defects is closely related to the timing of scanning and the experience of the ultrasonographer. The ability to image adequately fetal cardiac anatomy on the four chamber view increases rapidly when approaching 18 weeks' gestation. When ultrasonographers are taught to screen the four chamber view, this is obtained in $90 \%$ of examinations at 18 weeks' gestation or more, compared with only about $10 \%$ of examinations before 18 weeks' gestation. ${ }^{5}$ About half of our patients had a solitary routine scan before 18 weeks' gestation. Ideally, routine fetal anomaly scanning would be offered at 18 to 20 weeks' gestation, at a time when adequate visualisation is possible while still providing time for appropriate counselling should an important anomaly be detected. Funding for training programmes for ultrasonographers would be required. There is as yet no estimate of cost, but there would be savings in terms of reduced expenditure in the diagnosis, support, and management of children with serious cardiac defects as many of these parents would elect termination of pregnancy. There has been a marked reduction in the incidence of new patients with hypoplastic left heart syndrome at our supraregional centre over the last three years (45 in 1985-7 compared with 23 in 1988-90) which coincided with an increased rate of prenatal detection. ${ }^{8}$ In addition, the increased diagnosis of extracardiac anomalies that would occur with such a programme would almost certainly lead to a postnatal reduction in the incidence of such defects if parents terminate pregnancy in severe cases.

It is feasible to include the four chamber view of the fetal heart in a screening programme for the detection of fetal anomalies. The main drawback is that not all severe CHD will be detected by the four chamber screening view. It is not realistic to include more detailed examination of the fetal heart in a screening programme at present. Our choice of abnormalities which would be detectable on four chamber screening (table) was arbitrary, but intentionally conservative. Experience suggests that ultrasonographers who gain experience of four chamber fetal heart screening are increasingly likely to detect many abnormalities which we categorised as not detectable, including abnormalities of the arterial pole of the fetal heart.

It could be argued that the high proportion of our study group who would have elected termination of pregnancy had CHD been detected sufficiently early in pregnancy is misleading, as the information was obtained often at an emotionally difficult time and without the counselling which is obligatory when fetal anomalies are detected. On the other hand, these parents were experiencing the real consequences of congenital heart defects in their offspring and we were scrupulous not to lead them on this issue. At present, most mothers opt for termination of pregnancy if a severe fetal heart abnormality is detected. ${ }^{8}$ The opportunity to consider termination of pregnancy is not the only benefit of prenatal diagnosis, however, and the proportion of parents choosing this option may decrease as surgical results continue to improve. For some lesions, the major continuing mortality may be preoperative, ${ }^{9}$ and this is potentially avoidable if prenatal diagnosis occurs. Even neonatal heart transplantation has a higher likelihood of success with prenatal diagnosis as there is a longer time available for donor organ procurement.

\section{CONCLUSIONS}

Despite the widespread application of routine fetal ultrasound scanning in local hospitals, only a small percentage of the potentially detectable fetal cardiac defects are currently diagnosed. While the introduction of effective fetal cardiac screening would require major changes to current practice, it is feasible as part of a population based programme of fetal anomaly detection and could be expected to have a major impact on paediatric cardiology practice.

1 Joint study group on fetal abnormalities. Recognition and management of fetal abnormalities. Arch Dis Child 1989;64:971-6.

2 Allan LD. Diagnosis of fetal cardiac abnormalities. Arch Dis Child 1989;64:994-8.

3 Fermont L, de Geeter B, Aubry MC, Kachaner J, Sidi D. A close collaboration between obstetricians and pediatric cardiologists allows antenatal detection of severe cardiac malformations by two dimensional echocardiography. In: malformations by two dimensional echocardiography. In: Talner NS, eds. Pediatric cardiology. New York: Springer Talner NS, eds. Pedid

4 Allan LD, Crawford WC, Chita DC, Tynan MJ. Prenatal screening for congenital heart disease. BMF 1986;292: 1717

5 Sharland GK, Allan LD. Screening for congenital heart disease prenatally. Results of a 2.5 year study in the South East Thames region. Brf Obstet Gynaecol 1992; 99: 220-5. 6 Office of Population Censuses and surveys. 1989 Birth statistics. Series FMI No 18. London: HMSO, 14.

7 Anonymous. BMUS rejects call for more fetal heart scans. Radiography Today 1991; June:4.

8 Allan LD, Cook A, Sullivan I, Sharland G. Hypoplastic left heart syndrome - effects of fetal echocardiography on birth prevalence. Lancet 1991;337:959-61.

9 Rubay J, de Leval MR, Bull C. To switch or not to switch: the Senning alternative. Circulation 1988;78(suppl III): $1-4$ 